\title{
Estimación del descarte por exceso de captura en la pesquería industrial de cerco del stock Norte-Centro de la anchoveta peruana Engraulis ringens a partir de un programa de observación a bordo
}

\section{Estimating discard excess catch in the industrial purse seine fisheries of the North-Center stock of the Peruvian anchoveta (Engraulis ringens) from an on-board observer program}

\author{
Josymar Torrejón-Magallanes ${ }^{1 *}$, Elena Solana-Arellano ${ }^{2}$, Michel Dreyfus-León ${ }^{3,4}$
}

1 Instituto del Mar del Perú, esquina Gamarra y General Valle S/N, Chucuito, Callao, Perú.

2 Centro de Investigación Científica y Educación Superior de Ensenada (CICESE), Carretera Ensenada-Tijuana No. 3918, Zona Playitas, C.P. 22860, Ensenada, B.C. México.

3 Centro Regional de Investigación Pesquera, INAPESCA, Carretera Ensenada-Tijuana km 97.5, Parque Industrial FONDEPORT, EI Sauzal de Rodríguez, C.P. 22760, Ensenada, B.C. México.

4 Universidad Autónoma de Baja California (UABC), Carretera Transpeninsular Ensenada - Tijuana No. 3917. Colonia Playitas C.P. 22860

Ensenada, Baja California, México.

*Autor para correspondencia

Email Josymar Torrejón-Magallanes: ejosymart@gmail.com

Email Elena Solana Arellano: esolana@cicese.mx

Email Michel Dreyfus-León: dreyfus@cicese.mx

\begin{abstract}
Resumen
En este trabajo se presenta la estimación del descarte por exceso de captura en la pesquería industrial de cerco del stock Norte-Centro de anchoveta peruana. Se define el descarte por exceso de captura, como la porción de captura que se arroja al mar cuando se ha capturado más de lo que la capacidad de bodega de la embarcación puede almacenar. El análisis de estimación para el periodo 2005 - 2014, se realizó a partir del "Programa de observadores a bordo de la flota de cerco" que ejecuta el Instituto del Mar del Perú (IMARPE), en donde 5837 viajes, que representan el 1.6\% de los viajes totales, fueron muestreados. Las metodologías utilizadas en la estimación fueron: Modelo Lineal Generalizado (GLM) y el Modelo Delta. Las estimaciones por el Modelo Delta y el GLM fueron diferentes en magnitudes pero similares en tendencias, sin embargo la evaluación del funcionamiento del Modelo Delta, indica que este modelo se ajusta mejor a los datos. El Modelo Delta estimó que la pesquería descarta por exceso de captura entre 2954 y 199164 toneladas, con un promedio de 121312 toneladas para el periodo de estudio, el cual representó una tasa del $2.6 \%$ en relación a los desembarques. Se sugiere la incorporación de variables espaciales (p. ej. distancia a la costa, latitud, longitud), físicas (TSM) y del comportamiento del cardumen (p.e. agregación), para mejorar el análisis y comprender mejor el comportamiento de este tipo de descarte.
\end{abstract}

Palabras clave: Descarte; descarte por exceso de captura; anchoveta; modelo delta; modelo general lineal; programas de observación a bordo.

\section{Citación:}

Torrejón-Magallanes J., E. Solana-Arellano, M. Dreyfus-León. 2016. Estimación del descarte por exceso de captura en la pesquería industrial de cerco del stock Norte-Centro de la anchoveta peruana Engraulis ringens a partir de un programa de observación a bordo. Revista peruana de biología 23(2): 183 - 194 (Agosto 2016). doi: http://dx.doi.org/10.15381/ rpb.v23i2.12435

$\begin{array}{ll}\text { Presentado: } & 16 / 02 / 2016 \\ \text { Aceptado: } & 25 / 06 / 2016\end{array}$

Aceptado: 25/06/2016

Publicado online: $27 / 08 / 2016$
Información sobre los autores:

EJT realizó la redacción, obtención, análisis, y procesamiento de los datos. ESA realizó revisión y análisis de datos. MDL realizó revisión y análisis.

Los autores no incurren en conflictos de intereses.

Fuentes de financiamiento:

IMARPE - Proyecto de "Evaluación Indirecta de los Principales Recursos Pelágicos", Derechos de Pesca para la actividad de "Observación y evaluación en tiempo real del subsistema pelágico del Ecosistema de la corriente de Humboldt utilizando como plataforma la flota de cerco. 


\begin{abstract}
This paper presents estimates of the discard excess catch in the industrial purse seine fisheries of the NorthCenter stock of the Peruvian anchoveta (Engraulis ringens). Discard excess catch is defined as the portion of the catch that is thrown into the sea when has captured more than the hold capacity can store. The analysis is based on the "On-board observer of the purse seine vessels program" led by the Instituto del Mar del Peru (IMARPE), and in the period $2005-2014,5837$ trips were sampled, corresponding to $1.6 \%$ of the total trips. The methodologies used in the discard excess catch estimation were: Generalized Linear Model (GLM) and Delta Model. The estimations showed difference in magnitudes but similar trends, although the delta model appears to be a better alternative procedure for estimating the discard excess catch. The Delta Model estimated the purse seine fisheries discarded a range from 2954 to 199164 tons, an average estimated 121312 tons, which represent $2.6 \%$ of the landings in the period 2005 - 2014. Spatial variables (e.g. distance from the shore latitude, longitude), physical (SST), and school behavior (e.g. fish aggregation) should be included to improve the analysis and a better understanding of the discard excess catch behavior.
\end{abstract}

Keywords: Discard; discard excess catch; anchoveta; delta model; generalized linear model; On-board observer program.

\section{Introducción}

En ciencia pesquera, la captura incluye todo material biológico vivo el cual está representado por los desembarques y lo descartado (Viana 2012). El descarte es definido como la porción de captura que es devuelta al mar (Alverson et al. 1994). Se conocen diversos motivos por los que esta acción es realizada; así Clucas (1997) ha señalado los siguientes: 1) peces capturados que no pertenecen a la especie, talla o sexo objetivo, 2) el recurso se deteriora rápidamente luego de ser capturado, 3) falta de espacio en la embarcación, 4) cuotas alcanzadas, 5) captura de especies prohibidas, 6) captura en temporadas, áreas o utilizando artes de pesca prohibidas.

En décadas recientes ha crecido el interés por buscar soluciones al problema de realizar la acción del descarte, con el fin de establecer estrategias de manejo integral y holístico de los recursos y del ecosistema. La FAO, a través de su código de conducta para la pesca responsable (FAO 1995), fomenta el establecimiento y aplicación de políticas para la conservación de los recursos pesqueros, resaltando entre ellos que el descarte debe ser desalentado. Además, indica que se deben establecer programas de observación e inspección con la finalidad de reunir información confiable y precisa, en donde se incluya la captura incidental y los descartes. Esto último, es debido a que el descarte da cuenta de una proporción de la mortalidad por pesca $(F)$ que no es cuantificada, y se conoce que las conclusiones derivadas de la evaluación de stocks son claramente afectadas por la disponibilidad y confiabilidad de la información sobre las cantidades de peces que son descartados (Machias et al. 2001). Además, el descarte conduce a dos problemas fundamentales: una merma en el crecimiento y reclutamiento, lo que resulta en la reducción de los niveles poblacionales de las especies objetivo y no objetivo (Viana 2012). En este sentido, la estimación del descarte resulta esencial para poder evaluar el impacto de las pesquerías sobre la población de peces y el ecosistema sobre el cual ellos operan (Borges et al. 2005).

Los programas de observación a bordo constituyen una buena plataforma para obtener información acerca de las actividades pesqueras, incluyendo los volúmenes de descartes
(Babcock et al. 2003). Sin embargo, a pesar de su importancia, los volúmenes de descartes no siempre pueden ser incorporados a los procesos de evaluación de stocks, porque generalmente la información del descarte no es completa (p.e. años o meses no muestreados) debido a que estos programas se enfrentan a la falta de colaboración por parte de las empresas pesqueras para colocar a un observador a bordo de una embarcación, al bajo presupuesto designado para estas actividades, entre otros. En el Perú, el "Programa de observadores a bordo de la flota de cerco" es ejecutada por el Instituto del Mar del Perú y tiene como una de sus actividades el recolectar información de los descartes, los cuales junto a otras variables: espaciales, temporales y de características de las embarcaciones constituyen importantes insumos para modelar y estimar los descartes, pues se ha demostrado que estimaciones confiables de la captura total (el cual debe incluir a los descartes) para la evaluación de stocks, pueden ser obtenidos a partir de los datos que se colectan en este tipo de programas (Rochet et al. 2002).

Los primeros trabajos de estimación global de descarte, se basaron en información proveniente de literatura científica, informes y literatura "gris" disponible dentro de la FAO y desde contactos con expertos en administración pesquera e instituciones de investigación. Así la primera estimación de los descartes a nivel mundial fue realizada por Saila (1983), quien en un estudio para la FAO, mediante una revisión de las magnitudes de las capturas descartadas de las principales pesquerías alrededor del mundo, estimó un descarte mínimo de 6.72 millones de toneladas anuales, el cual incluyó sólo peces e invertebrados. Alverson et al. (1994) en un estudio global de los descartes para la pesquería comercial, propusieron una metodología de estimación, en la cual señalan que el descarte está en función de los desembarques de la especie objetivo, obteniendo un estimado entre 17.9 - 35 millones de toneladas (promedio de $27 \mathrm{Mt}$ ). Así mismo, Kelleher (2008) propuso un enfoque alternativo para la estimación global de los descartes, donde asume que el descarte está en función de la pesquería (área, equipo de pesca, especie objetivo) y estimó una magnitud global de descarte de 6.8 millones de toneladas. Además en este trabajo resalta, que es necesario contar con un programa de observación para ob- 
tener una estimación precisa del descarte, en la cual se requiere un muestreo exhaustivo, pues la metodología de extrapolación (usada en estos trabajos) depende mucho de las muestras tomadas por los observadores en estos programas.

Para la pesquería de anchoveta peruana, el primer y único estudio enfocado en describir y estimar el descarte fue realizado por Castillo y Mendo (1987) quienes señalaron que existen diferentes fuentes de descarte: el descarte por exceso de captura, la pérdida de fluidos, el pesaje fraudulento, el descarte de juveniles y pesca negra (captura de otras especies que son vendidas como anchoveta). De los componentes mencionados, el descarte por exceso de captura, el cual podemos definir como la porción de captura que se arroja al mar cuando se ha capturado más de lo que la capacidad de bodega de la embarcación puede almacenar, representó en el trabajo de Castillo y Mendo (1987) uno de los mayores porcentajes con respecto a los desembarques con un 9.13\%. Sin embargo, este estudio estuvo restringido al área entre Chicama $\left(7^{\circ} \mathrm{S}\right)$ - Callao $\left(12^{\circ} \mathrm{S}\right)$ y no abarcó toda la extensión de distribución del stock Norte-Centro de anchoveta peruana. Además la metodología de estimación, estuvo basada en encuestas al personal relacionado con la pesca de anchoveta (miembros de tripulación, personal administrativo y empleados de las plantas procesadoras de harina) cuyas preguntas estaban enfocadas a conocer la cantidad del descarte en sus diferentes fuentes.

En diferentes pesquerías alrededor del mundo se han empleado varias metodologías para estimar los descartes a partir de la información colectada en los programas de observación; entre ellas se mencionan los modelos lineales generalizados (GLM), estimación por estratificación o utilizando ponderación mediante proporciones (p.e. Ortiz et al. 2000, Rochet et al. 2002, Borges et al. 2005, Lema et al. 2006). La estimación por GLM presenta varias ventajas respecto a los otros métodos mencio-

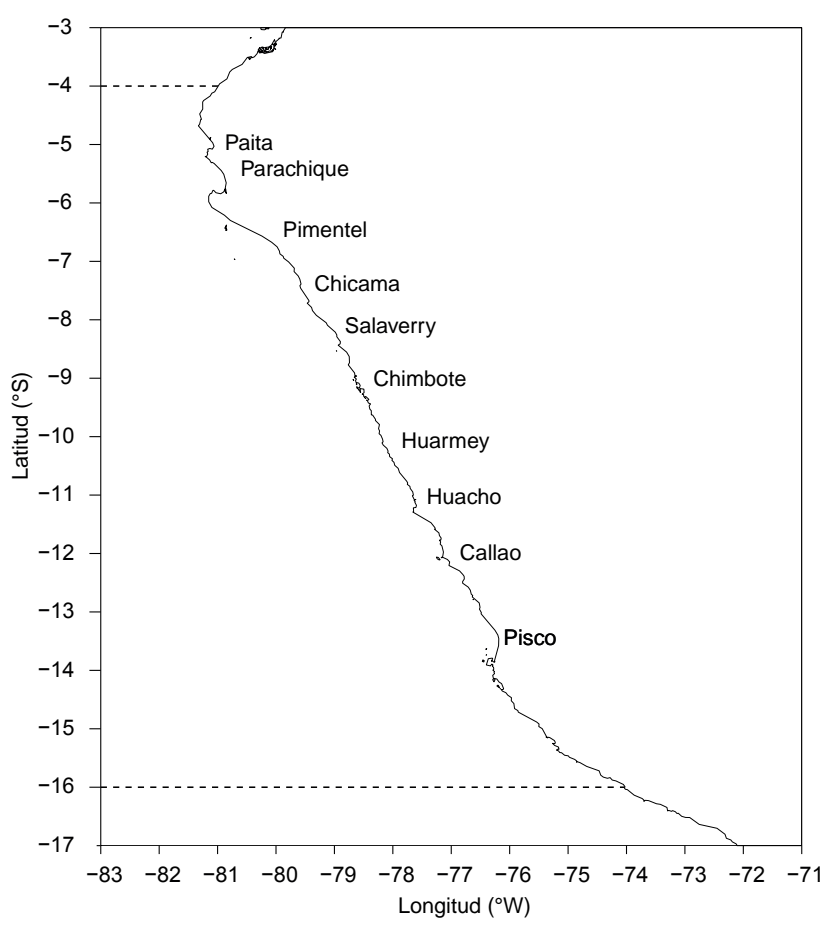

Figura 1. Zona de distribución donde opera la flota industrial de cerco orientada a la pesca del stock Norte - Centro de anchoveta peruana $\left(4^{\circ} \mathrm{S}-16^{\circ} \mathrm{S}\right)$. (Línea punteada) nados: permite cuantificar las relaciones entre un conjunto de variables continuas y/o categóricas, es flexible a la distribución de datos pues involucra una variedad de distribuciones seleccionadas de una familia exponencial especial (Lindsay 1997) y utiliza el método de máxima verosimilitud para la estimación de parámetros (Cordeiro \& Demetrio 2000). Otra metodología que ha sido utilizada para estimar el descarte es el Modelo Delta (p.ej. Ortiz et al. 2000), particularmente útil cuando se tienen observaciones con muchos ceros (Pennington 1983, Lo et al. 1992). Este modelo se compone de dos sub-modelos: a) Aquel que modela la probabilidad de tener observaciones que no son ceros y b) aquel que modela la probabilidad de obtener cierto volumen descartado si hay una observación positiva de descarte (Ortiz et al. 2000). La ventaja de su formulación radica en que cada sub-modelo puede ser expresado en términos de un GLM.

El objetivo de este estudio es estimar la magnitud del descarte por exceso de captura en la pesquería industrial de cerco del stock Norte-Centro de la anchoveta peruana a partir de los datos proporcionados por el "Programa de observadores a bordo de la flota de cerco" y comparando 2 metodologías: el GLM y el Modelo Delta. Se discute la eficacia de estos métodos para saber qué metodología se adapta a los datos y realiza estimaciones más precisas. Los resultados permitirán hacer proyecciones más confiables y útiles para la evaluación y manejo del stock y a la definición de políticas que garanticen óptimos niveles de explotación.

\section{Material y métodos}

Periodo y área de estudio.- El periodo de estudio constó de 10 ańos (2005 - 2014) y abarcó la zona de distribución del stock Norte-Centro de la anchoveta peruana $\left(4^{\circ} \mathrm{S}-16^{\circ} \mathrm{S}\right)$ (Fig. 1). En esta zona se encuentra aproximadamente el $89 \%$ de la biomasa total de anchoveta con un desembarque anual del $87 \%$ y opera el 90\% de la flota (Fréon et al. 2008). Durante el tiempo de estudio en esta área de distribución, se observaron 5837 viajes de un total de 371468 (Tabla 1). El mayor esfuerzo de muestreo, el cual se refiere al porcentaje de viajes observados, ocurrió en los años 2005-2008 y 2013, decayendo en los demás años,

Tabla 1. Viajes observados por el "Programa de observadores a bordo de la flota de cerco", viajes totales en la pesquería industrial de cerco del stock Norte-Centro de la anchoveta peruana (2005 - 2014) y el porcentaje de cobertura observada.

\begin{tabular}{cccc}
\hline Año & $\begin{array}{c}\text { Viajes } \\
\text { observados }\end{array}$ & $\begin{array}{c}\text { Viajes } \\
\text { totales }\end{array}$ & $\begin{array}{c}\text { Cobertura } \\
\text { observada }(\%)\end{array}$ \\
\hline 2005 & 1016 & 57451 & 1.8 \\
2006 & 721 & 38917 & 1.9 \\
2007 & 738 & 37774 & 2.0 \\
2008 & 699 & 44126 & 1.6 \\
2009 & 435 & 41605 & 1.0 \\
2010 & 75 & 23341 & 0.3 \\
2011 & 370 & 49119 & 0.8 \\
2012 & 270 & 29494 & 0.9 \\
2013 & 1023 & 34522 & 3.0 \\
$2014^{*}$ & 491 & 15329 & 3.2 \\
\hline
\end{tabular}

* Solo una temporada de pesca 
especialmente en el 2010 donde se registró el menor porcentaje, esto se debió al bajo presupuesto asignado al programa ese año.

\section{Estimación del descarte por exceso de captura}

Modelo Lineal Generalizado (GLM).- La variable respuesta es el descarte por exceso de captura (dex) por viaje. En el modelo, está en función lineal del año, mes, temporada de pesca, puerto y capacidad de bodega (cb). Antes de incluirla en el modelo, a la variable dex se le sumó una constante positiva $c$ y se le aplicó la transformación logaritmo natural. La suma de $c$ se realizó para evitar problemas de resultados indefinidos al momento de aplicar logaritmos a valores que son ceros. La transformación logarítmica se hizo para alcanzar la normalidad en los datos transformados (Berry 1987). El modelo es definido como:

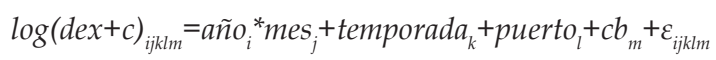

Donde,

dex: el descarte por exceso de captura que se realiza en un viaje de pesca.

año, mes, temporada, ${ }_{i}$ puerto, caracteriza la variabilidad espacio temporal en la pesca de anchoveta, $c b_{m}$ hace referencia a la capacidad de bodega del barco y,

$\varepsilon_{i j k l m}:$ El error, el cual se asume aleatorio, independiente y normalmente distribuido $N(0,1)$.

El dex predicho para cada estrato $(i, j, k, l, m)$ se estimó utilizando la inversa del logaritmo natural y restando la constante c. Además, si los supuestos sobre la distribución del error son correctos, ocurre un error sistemático el cual puede compensarse usando el factor de corrección Sprugel (1983):

$$
\left.\widehat{d e x_{i j k l m}}=e^{\left(\hat{Y}_{i j k l m}+0.5 * \sigma_{\varepsilon_{\hat{Y}} j k m}^{2}\right.}\right)-c
$$

Donde,

$\hat{Y}_{i, k h m}: \log (\operatorname{dex}+\mathrm{c})$ predicho por el GLM para los estratos $i, j, k, l, m$.

$\sigma_{\tilde{\varepsilon}_{\hat{Y}_{i j k l m}}}$ : la varianza de los residuales del GLM o el cuadrado medio de los residuales.

El dex predicho en cada estrato se multiplicó por el número de viajes realizados por la flota industrial de cerco dedicada a la pesca de anchoveta peruana en dicho estrato. El dex total estimado por año se calculó como la suma del dex en todos los estratos para el año $i$ :

$$
\operatorname{dex}_{i}=\sum_{j k l m} \widehat{\operatorname{dex}}_{i j k l m}{ }^{*} f_{i j k l m} \ldots \ldots \ldots . . .(\text { Ec. } 3)
$$

Donde,

$f_{i j k l m}$ es el esfuerzo total en número de viajes totales realizados por toda la flota dedicada a la pesca de anchoveta peruana para el año $i$, mes $j$, temporada $k$, puerto $l, \mathrm{cb} m$.

En este trabajo se presentan 3 casos para estimar los descartes mediante el GLM y ver el efecto sobre la estimación al usar diferentes valores de la constante. Usamos como constante los valores: 1, el mínimo valor del descarte positivo (min dpue>0) y 0.5, este último sugerido por Yamamura (1999) para estabilizar la varianza. El porcentaje de explicación de cada modelo se basó en el cambio de la devianza residual, la cual es el equivalente al coeficiente de determinación $\mathrm{R}^{2}$ (Stefánsson 1996). Además se evalúa, cuál es la influencia en las estimaciones al no considerar ciertos factores en el GLM (se toma de base el caso $c=1$ ).

Para las estimaciones, la costa peruana fue dividida en 7 grupos de puertos que para simplificar llamaremos Puertos. Así tenemos: Puerto 1: Bayovar - Paita - Parachique, Puerto 2: Chicama - Salaverry, Puerto 3: Casma - Coishco - Chimbote, Puerto 4: Huarmey, Puerto 5: Chancay - Huacho - Supe, Puerto 6: Callao, Puerto 7: Pisco - Tambo de Mora.

Además, la capacidad de bodega de los barcos fue dividida en 6 grupos; $\mathrm{CB} 1 \leq 100$ t., $100<\mathrm{CB} 2 \leq 200$ t., $200<\mathrm{CB} 3 \leq$ 300 t., $300<\mathrm{CB} 4 \leq 400$ t., $400<\mathrm{CB} 5 \leq 500$ t., CB6 > 501 t.

Modelo Delta.- Los modelos Delta son usados particularmente cuando se tienen observaciones con muchos ceros (Pennington 1983, Lo et al. 1992). Para este tipo de distribución generalmente se usan transformaciones con la finalidad de que los datos cumplan con los supuestos de normalidad, ya que en casos en los cuales la proporción de ceros es muy alta, los estimadores de tendencia central resultan estar sesgados, con baja precisión y con una varianza sobreestimada (Pennington 1996).

En un modelo delta, los valores estimados son el producto de dos componentes independientes o sub-modelos: a) La probabilidad de tener observaciones que no son ceros y b) la probabilidad de obtener un cierto valor si hay una observación positiva (Ortiz et al. 2000). El primer sub-modelo asume una distribución binomial de los errores, mientras que el segundo puede ser basado en diferentes distribuciones de error como lognormal, gamma, Poisson, entre otros (Ortiz \& Arocha 2004). La ventaja de su formulación radica en que cada componente del modelo delta puede ser expresado en términos de un GLM (McCullagh 1984).

El modelo delta genera estimaciones anuales de los descartes. Estas estimaciones se obtienen mediante el producto de tres componentes: 1) la proporción de viajes con descarte positivo $p, 2)$ la tasa media de descarte (por exceso de captura) por viaje si hay una observación positiva de descarte y 3) el número de viajes realizado por toda la flota industrial de cerco dedicada a la pesca de anchoveta.

Proporción de viajes con descarte positivo (p).- Para hacer esta estimación se clasificó a los viajes sin descarte por exceso de captura como 0 y a los que presentaron descarte positivo como 1, con lo que tenemos una distribución del tipo binomial. Esta distribución está definida en términos de la proporción (y) de los viajes con descarte positivo $(r)$ con respecto al total (n). La función de densidad $f(y)$ y la función de varianza asociada $\operatorname{Var}(y)$ está dada por:

$$
\begin{aligned}
& y=\frac{r}{n}, f(r)=\left(\begin{array}{l}
n \\
r
\end{array}\right) \mu^{r}(1-\mu)^{n-r} \\
& \text { donde: } \quad r=1,2,3, \ldots
\end{aligned}
$$

$$
\operatorname{Var}(y)=\frac{\mu(1-\mu)}{n}
$$

donde $\mu=$ media de $y$

El predictor lineal de la proporción de los descartes será una función lineal de las variables explicativas año, mes, puerto, 
capacidad de bodega $(c b)$ así:

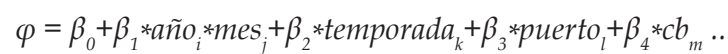

Donde,

$\beta_{i}$, son los parámetros a ser estimados.

Tasa media de descarte.- Para esta parte de la estimación, solo se consideró aquellos viajes con descarte mayor a 0 toneladas (positivos). Se utilizó como variable respuesta el descarte por exceso de captura (dex) el cual tiene una resolución por viaje. La media del dex dado un descarte positivo fue estimado siguiendo un GLM. Para la normalización de nuestra variable dependiente que llamaremos ahora $b c(d e x)$, se utilizó la familia de transformaciones Box - Cox. Este procedimiento planteado por Box y Cox (1964) donde lambda $(\lambda)$ es un parámetro a estimar, ha sido utilizado en muchos trabajos para resolver los problemas de falta de normalidad y de homocedasticidad (Guerrero \& Johnson,

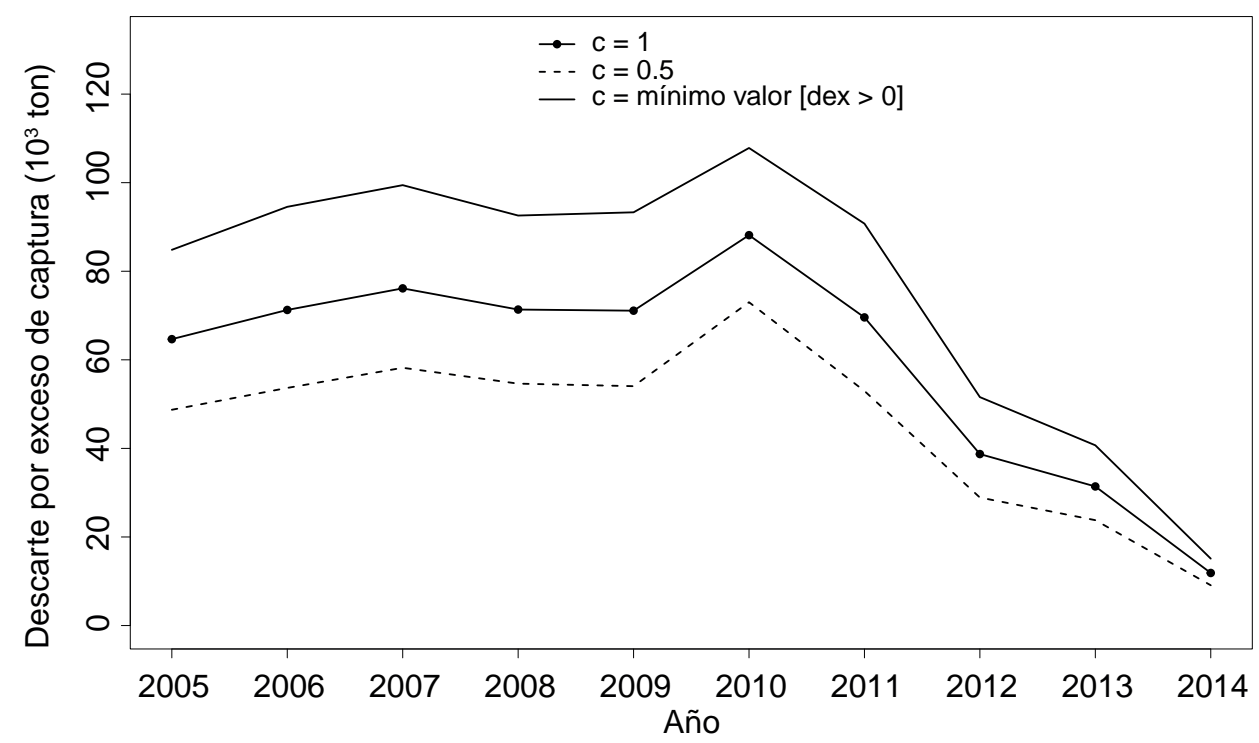

Figura 2. Estimaciones del descarte por exceso de captura en la pesquería industrial de cerco del stock Norte-Centro de la anchoveta peruana mediante el GLM con diferentes valores de la constante $c$.

Tabla 2. Análisis de la devianza para los ajustes mediante el GLM sumando diferentes valores de $c$ a la variable dependiente.

\begin{tabular}{|c|c|c|c|c|c|}
\hline Factor & Df & Devianza residual & Cambios en devianza & $\%$ de devianza total & $p$-valor \\
\hline \multicolumn{6}{|c|}{$c=1$} \\
\hline & & 6905.3 & & & \\
\hline Temporada & 1 & 6891.3 & 14.0 & 0.20 & $<0.05$ \\
\hline Año & 9 & 6695.8 & 195.5 & 3.03 & $<0.05$ \\
\hline Mes & 7 & 6646.7 & 49.1 & 3.74 & $<0.05$ \\
\hline Puerto & 6 & 6520.2 & 126.5 & 5.58 & $<0.05$ \\
\hline Capacidad Bodega & 5 & 6487.8 & 32.4 & 6.05 & $<0.05$ \\
\hline Año:Mes & 38 & 6363.2 & 124.6 & 7.85 & $<0.05$ \\
\hline \multicolumn{6}{|c|}{$c=0.5$} \\
\hline & & 9365.1 & & & \\
\hline Temporada & 1 & 9346.7 & 18.4 & 0.20 & \\
\hline Año & 9 & 9077.9 & 268.8 & 3.07 & $<0.05$ \\
\hline Mes & 7 & 9011.4 & 66.5 & 3.78 & $<0.05$ \\
\hline Puerto & 6 & 8836.0 & 175.4 & 5.65 & $<0.05$ \\
\hline Capacidad Bodega & 5 & 8792.8 & 43.2 & 6.11 & $<0.05$ \\
\hline Año:Mes & 38 & 8625.6 & 167.2 & 7.90 & $<0.05$ \\
\hline \multicolumn{6}{|c|}{$c=$ mínimo valor $[$ descarte por exceso $>0])$} \\
\hline & & 4875.1 & & & \\
\hline Temporada & 1 & 4864.8 & 10.3 & 0.21 & $<0.05$ \\
\hline Año & 9 & 4729.6 & 135.2 & 2.98 & $<0.05$ \\
\hline Mes & 7 & 4695 & 34.6 & 3.69 & $<0.05$ \\
\hline Puerto & 6 & 4608.4 & 86.6 & 5.47 & $<0.05$ \\
\hline Capacidad Bodega & 5 & 4585.2 & 23.2 & 5.95 & $<0.05$ \\
\hline Año:Mes & 38 & 4496.4 & 88.8 & 7.77 & $<0.05$ \\
\hline
\end{tabular}



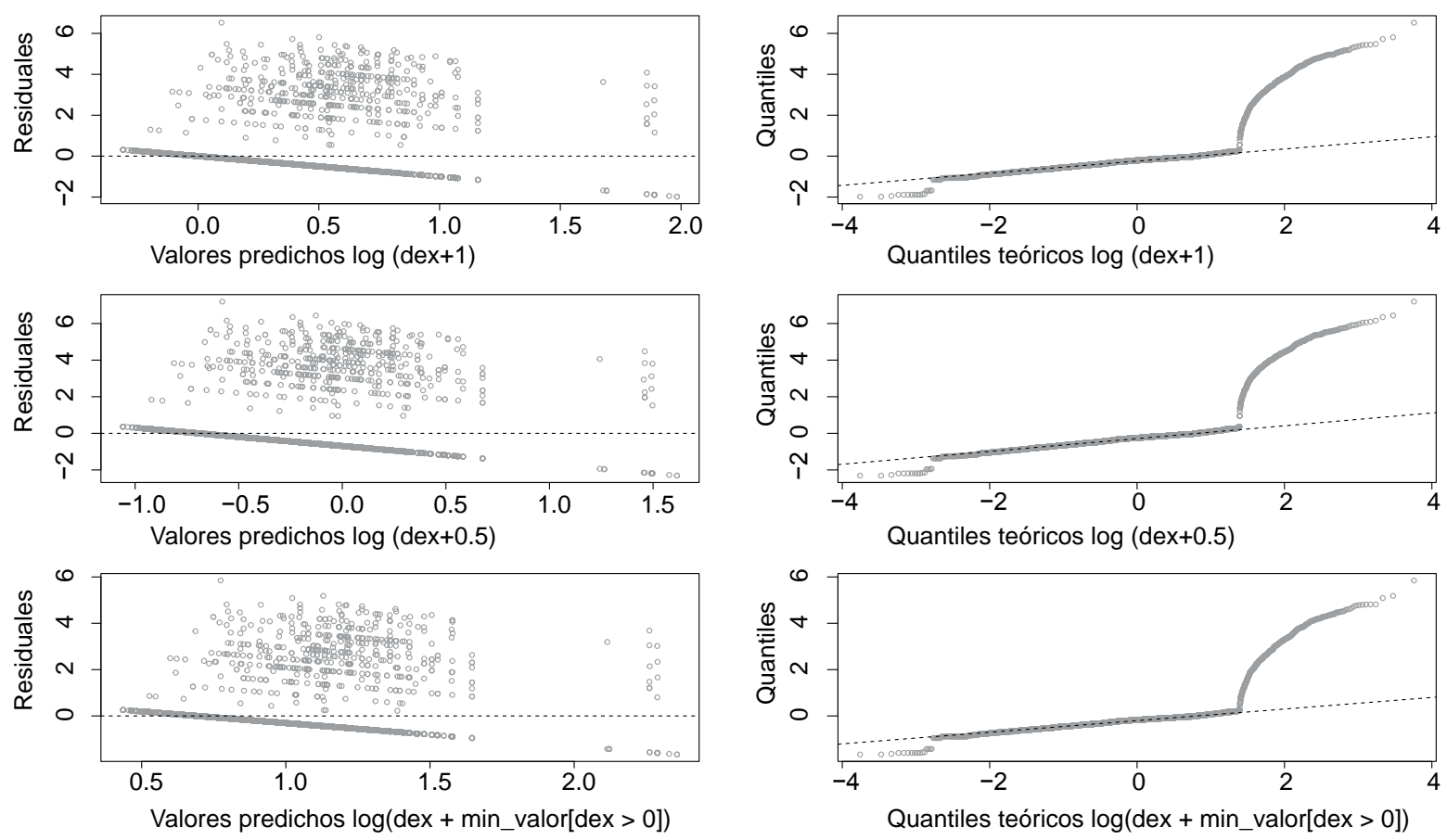

Figura 3. Gráficas de los residuales contra los valores predichos del descarte por exceso de captura con diferentes valores de la constante $c$.

1982, Spitzer 1982, Sakia, 1992). La transformación Box-Cox consiste en transformar cualquier variable con distribución desconocida a una distribución normal mediante la función:

$$
Y^{(\lambda)}= \begin{cases}\frac{Y^{\lambda}-1}{\lambda} ; & \lambda \neq 0, \\ \log (Y) ; & \lambda=0 .\end{cases}
$$

Donde,

$Y$ : es la variable con distribución desconocida y $\lambda$ es el parámetro normalizador

Luego el modelo de regresión queda definido como:

$b c($ dex $)=\beta_{0}+\beta_{1} * a \tilde{n} o_{i} *$ mes $+\beta_{2} *$ temporada ${ }_{k}+\beta_{3} *$ uerto $+\beta_{4} * c b_{m} \ldots$

Donde,

$b c($ dex) es el descarte por exceso de captura para los viajes con descarte mayor a 0 toneladas, a la cual se le hizo una transformación Box-Cox y $\beta_{i}$ son los parámetros a ser estimados.

Finalmente el descarte medio por año $(i)$ se calculó como el producto de la proporción de viajes con descarte positivo $\left(\hat{p}_{i j k l m}\right)$, la tasa media de descarte ( $\widehat{d e x}_{i j k l m}$ ) y el esfuerzo correspondiente $\left(f_{i j k l m}\right)$ el cual es número de viajes totales realizado por toda la flota industrial de cerco dedicada a la pesca del stock NorteCentro de la anchoveta.

$$
\text { Descarte }_{i}=\sum_{j k l m} \widehat{p}_{i j k l m} \widehat{* d e} x_{i j k l m}{ }^{*} f_{i j k l m} \ldots \ldots \ldots . . .(E c .8)
$$

Además, una vez obtenido el valor del descarte medio anual, se sumó este valor a los desembarques para obtener el valor de la captura anual corregida $\left(C_{c}\right)$, asumiendo que el descarte por exceso de captura es la única fuente de captura que no es reportada en la pesca.

$$
C_{c i}=\Sigma \text { Desembarque }_{i}+\text { Descarte por exceso }{ }_{i}
$$

\section{Resultados}

Modelo General Lineal. - Los porcentajes de variación explicada por cada modelo fueron bajos y similares entre sí, con valores cercanos al 8\% (Tabla 2). Las estimaciones anuales de los descartes mostraron una variación en la magnitud dependiendo del valor de la constante $c$ que es sumada, sin embargo las tendencias fueron las mismas (Fig. 2). Las estimaciones promedio para cada caso fueron de 59216, 45701 y 77068 toneladas.

Se muestra el análisis gráfico de cada caso para evaluar el comportamiento de la varianza en los residuales (Fig. 3), en la cual se observa que existe una tendencia en el valor de los residuales con respecto a los valores predichos. También se muestra el análisis de normalidad en los residuales (Fig. 3), en las cuales se observa que no se ajustan a la línea punteada. Además, con base en la estimación del descarte usando como valor de constante $c=1$, se observa que no difieren significativamente tanto en las estimaciones como en las tendencias aun cuando el modelo original (GLM) es modificado en su totalidad (Fig. 4).

Modelo Delta. - Los valores observados de la variable de respuesta no poseen una distribución normal y presentan algunos valores extremos. Además el gráfico de probabilidad normal muestra que la distribución de la variable respuesta posee colas más gruesas que el de la distribución normal (Fig. 5).

$\mathrm{Al}$ aplicar la transformación Box-Cox, el parámetro lambda $(\lambda)$ obtenido para dex, fue de 0.048 , por lo que la nueva variable resultó de aplicar la siguiente ecuación: 


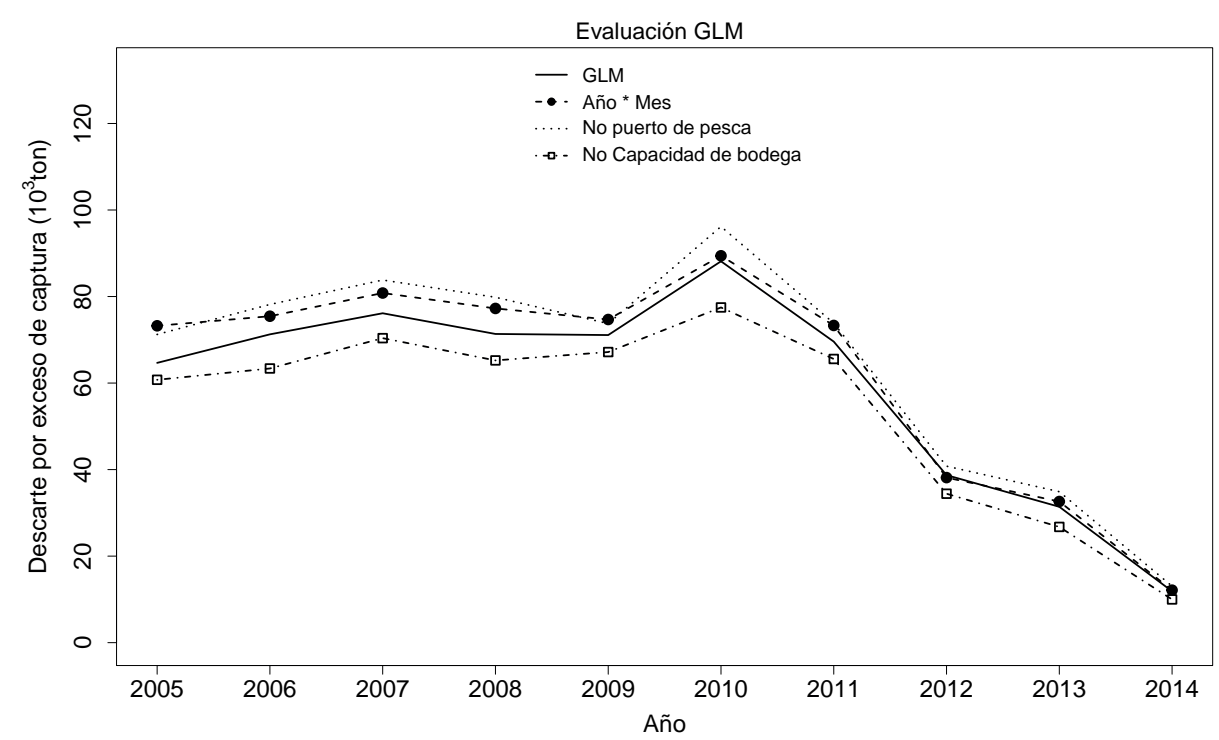

Figura 4. Estimación del descarte por exceso de captura con el modelo GLM y modelos modificados del GLM.
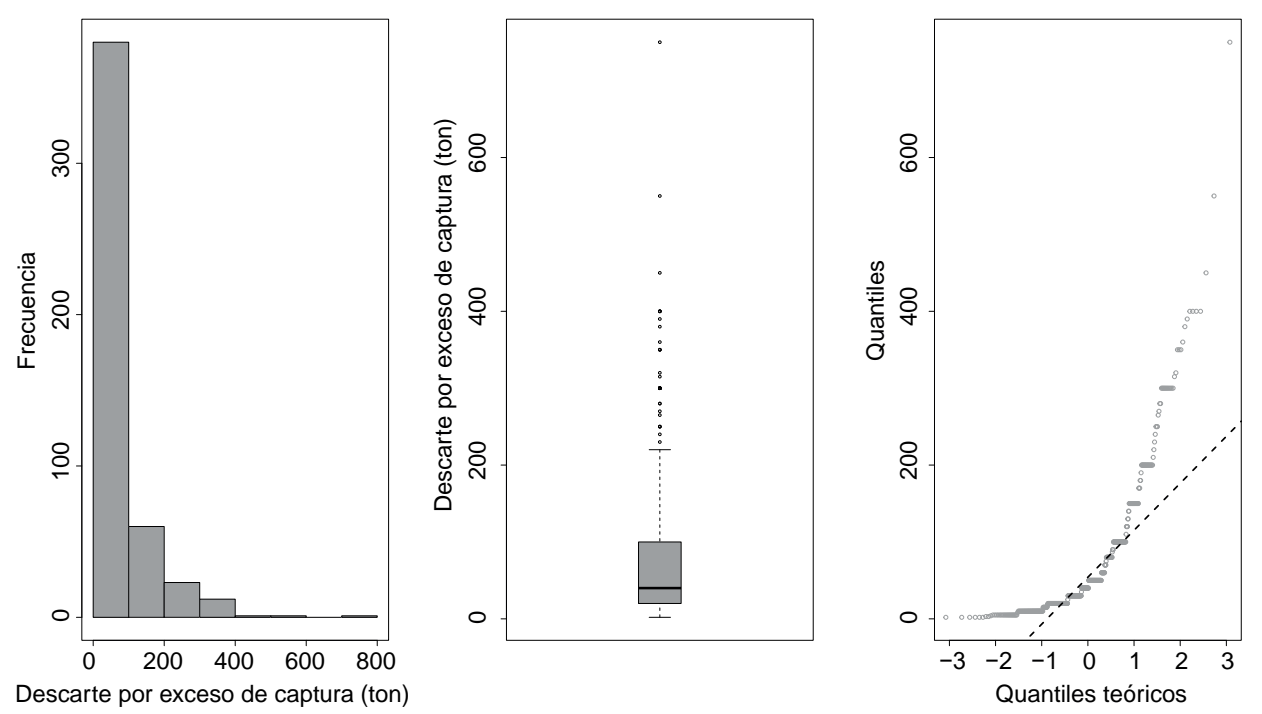

Figura 5. Análisis gráfico de los valores observados de la variable respuesta.
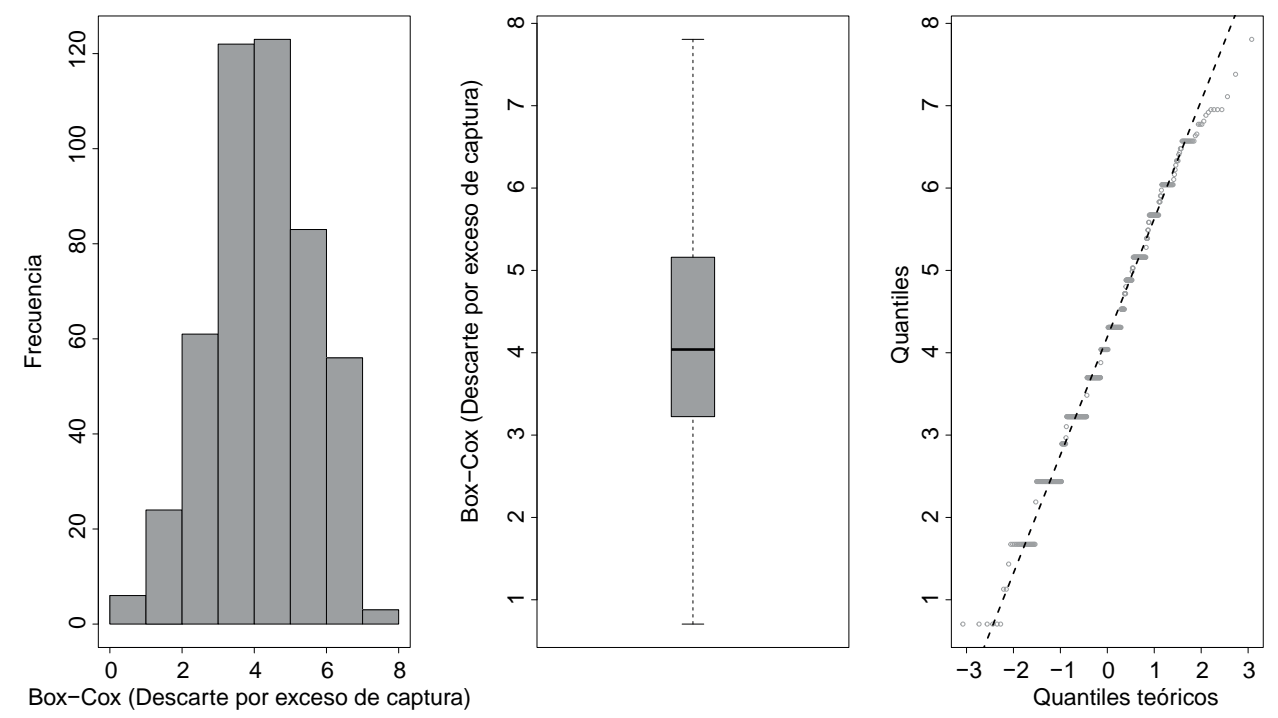

Figura 6. Análisis gráfico de los valores transformados de la variable respuesta. 

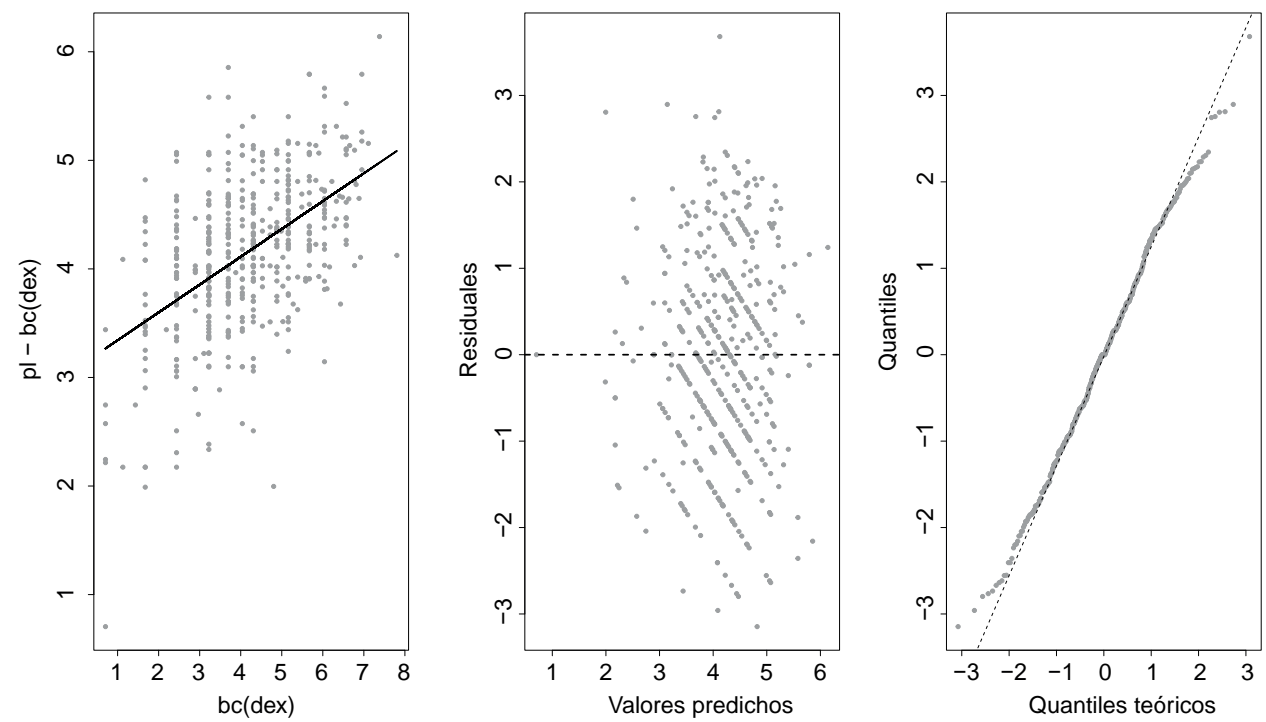

Figura 7. Predictor lineal ( $\mathrm{pl}$ ) estimado por el modelo contra la variable dependiente transformada. Residuales contra los valores predichos. $\mathrm{QQ}$-plot de los residuales para evaluar normalidad.

$$
b c\left(\operatorname{dex}_{i}\right)=\frac{\operatorname{dex}_{i}^{0.048}-1}{0.048}
$$

Con esta transformación la variable se acercó a la distribución normal, se llegó a una simetría en los datos, minimización de datos extremos y los residuales no presentaron ninguna tendencia marcada (Fig. 6).

Para evaluar el componente tasa media de descarte mediante el modelo delta, se utilizaron pruebas gráficas como la precisión de la función de enlace, precisión de varianza de residuales y su normalidad. El graficar la variable dependiente ajustada bc(dex), contra el predictor lineal estimado nos permite evaluar la función de enlace. En la Figura 7 se muestran las gráficas del predictor lineal ( $\mathrm{pl}$ ) contra la variable dependiente transformada (bc) para el descarte por exceso de captura (dex), las cuales fueron significativas $(\mathrm{p}<0.05)$ con un coeficiente de determinación $\left(\mathrm{R}^{2}\right)$ de 0.26 . El comportamiento de la varianza de los residuales fue evaluado usando un gráfico de residuales contra los valores predichos por el modelo, en la cual se espera que la dispersión de los residuales sea constante e independiente de los valores predichos. Es decir los residuales deben estar distribuidos alrede-

Tabla 3. Análisis de la devianza para los ajustes mediante el Modelo Delta.

\begin{tabular}{cccccc}
\hline Factor & Df & $\begin{array}{c}\text { Devianza Cambios en } \\
\text { residual }\end{array}$ & $\begin{array}{c}\text { \% de } \\
\text { devianza } \\
\text { devianza }\end{array}$ & $\begin{array}{c}p \text {-valor } \\
\text { total }\end{array}$ & \\
\hline & & 925.91 & & & \\
Temporada & 1 & 914.38 & 11.5 & 1.25 & $<0.05$ \\
Año & 9 & 861.65 & 52.7 & 6.94 & $<0.05$ \\
Mes & 6 & 847.44 & 14.2 & 8.47 & $<0.05$ \\
Puerto & 6 & 819.34 & 28.1 & 11.51 & $<0.05$ \\
Capacidad & 5 & 773.47 & 45.9 & 16.46 & $<0.05$ \\
Bodega & & & 84.9 & 25.63 & $<0.05$ \\
Año:Mes & 31 & 688.58 & 84.9 & & \\
\hline
\end{tabular}

dor del cero y no presentar una tendencia marcada con respecto a los valores ajustados. Una tendencia positiva (pendiente $>0$ ) indica que la función de varianza asumida aumenta conforme aumenta la media y viceversa (McCullagh 1984). Los resultados muestran que los residuales no presentan ninguna tendencia, por lo tanto son constantes e independientes con respecto a los valores ajustados (Fig.7). Del mismo modo una gráfica de probabilidad de residuales es usada para evaluar la normalidad en los residuales, en la cual se espera que exista una línea recta, especialmente cerca del centro; los resultados confirman la distribución normal de los residuales (Kolmorogov - Smirnov, $\mathrm{D}=0.031, \mathrm{p}=0.3112$ ) (Fig. 6). En la Tabla 3 se muestra el $\mathrm{R}^{2}$, la devianza residual, grados de libertad de los factores, y el p valor. Se observa que todas las variables fueron significativas y el porcentaje de variación explicada fue del $26 \%$.

La estimación anual para el descarte por exceso de captura tuvo un máximo en el 2006 con 199164 ton. y un mínimo el 2014 con 2954 ton. con un promedio de 121312 ton, además se presentan los intervalos de confianza (Fig. 8, Tabla 4).

\section{Discusión}

El análisis del descarte por exceso de captura muestra que la estimación mediante el GLM difiere en sus magnitudes mas no en las tendencias generadas por el Modelo Delta.

Al aplicar el GLM las estimaciones presentan sensibilidad, ya que el resultado depende de la constante que se le sume a la variable dependiente (Ec. 1). Así, mientras mayor sea el valor de la constante sumada, mayor será el valor de la estimación, sin embargo no siempre sucede este comportamiento. Ortiz et al. (2000) en un análisis para la estimación del bycatch en la pesquería de arrastre de camarón en el Golfo de México, utilizaron diferentes valores de constante en la transformación logarítmica de la variable respuesta, y demostraron que usar el menor valor de la constante, podría hacer que las estimaciones del bycatch sean mayores en comparación a cuando se usa una constante mayor. Estos cambios en la magnitud del descarte por exceso de captura, que dependen de la constante, estarían surgiendo debido a que los datos de descarte por exceso de 


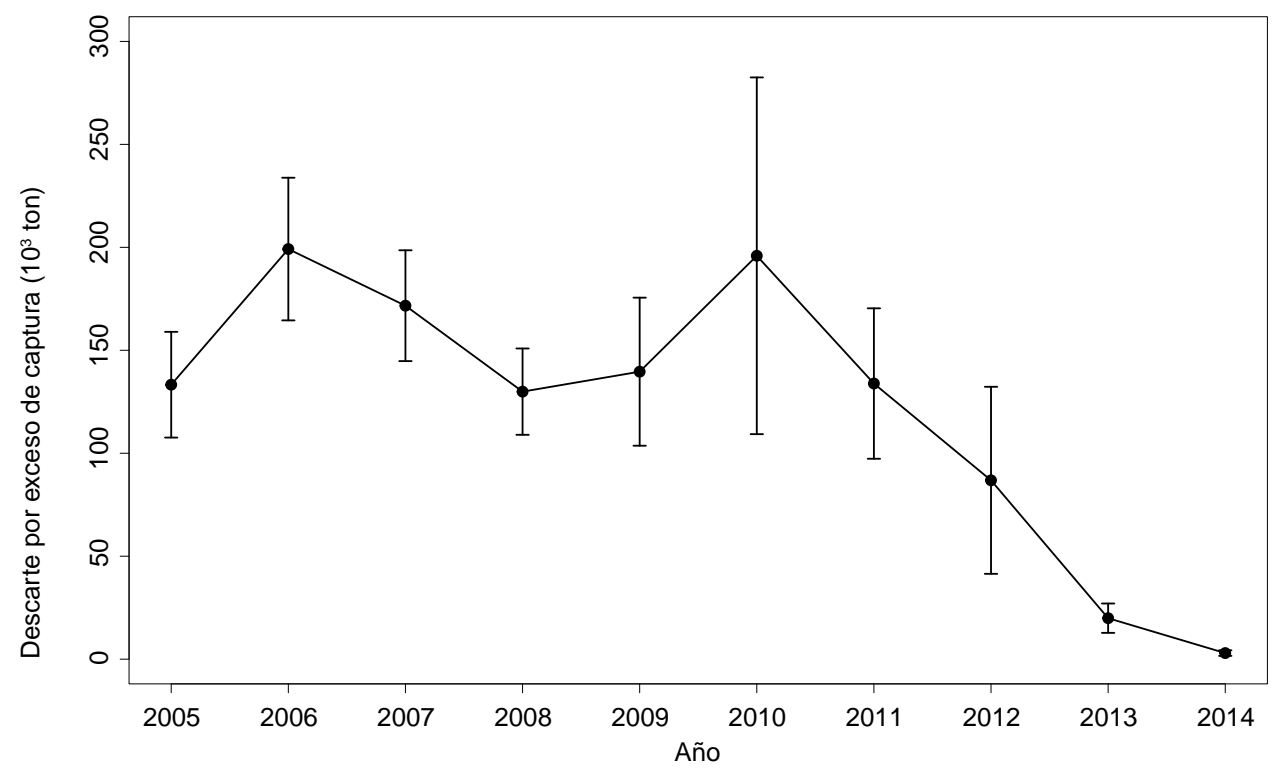

Figura 8. Estimación del descarte por exceso de captura en la pesquería industrial de cerco del stock Norte-Centro de la anchoveta peruana a partir del Modelo Delta.

captura no cumplen con los supuestos asociados con el GLM, pues las variables respuesta no presentan distribución lognormal debido a la gran proporción de ceros.

$\mathrm{Al}$ analizar la devianza del GLM para todos los casos ( $\mathrm{c}=$ $1, \mathrm{c}=5, \mathrm{c}=$ valor mínimo del descarte positivo observado) se obtuvieron bajos valores del coeficiente de determinación $\left(\mathrm{R}^{2}\right.$ $8 \%)$. Además en el análisis de influencia en las estimaciones al no considerar ciertos factores en el modelo (Fig. 4), los resultados mostraron que las estimaciones no varían sustancialmente. Estos resultados sugieren que en este modelo la temporada, puerto y capacidad de bodega son factores que no estarían contribuyendo significativamente a la explicación de la variabilidad observada en los datos. Aunque todas las variables fueron significativas ( $\mathrm{p}$ $<0.05$ ) en todos los escenarios, este puede ser una respuesta a la gran cantidad de grados de libertad de cada variables (factor). Entonces, es posible que la estructura del GLM no esté reflejando todos los principales factores que explican la variabilidad del descarte entre años. Por tanto, para este caso, el GLM que solo incluye las variables año y mes con su interacción produce estimados similares en relación al incluir todas las variables (Ec. 6).

Por otro lado, se evaluaron las estimaciones del GLM, a través de los supuestos que debe presentar la distribución de los residuales. El patrón esperado es una distribución de residuales con media cero y varianza constante. Las salidas del GLM se alejan totalmente del patrón esperado, la tendencia es claramente afectada por la presencia significativa de los ceros en los valores de descarte presentando una tendencia negativa. Esto indica que la varianza de esa distribución de error no toma en cuenta la disminución de la variabilidad observada, particularmente para los valores altos del descarte por exceso observado.

En cuanto al Modelo Delta se cumplieron todas las especificaciones, en consecuencia un análisis de devianza fue hecho para evaluar la importancia de los factores seleccionados en este modelo. Las variables ańo, mes, puerto, capacidad de bodega

Tabla 4. Estimación del descarte por exceso de captura en la pesquería industrial de cerco del stock Norte-Centro de la anchoveta peruana mediante el Modelo Delta. Se presenta además los intervalos de confianza del descarte por exceso de captura, los desembarques y la proporción del descarte con respecto a los desembarques.

\begin{tabular}{lcccc}
\hline Año & Descarte por exceso de captura (ton) & Intervalo de Confianza & Desembarque (ton) & Proporción (\%) \\
\hline $\mathbf{2 0 0 5}$ & 133290 & $107614-158966$ & 7544064 & 1.8 \\
$\mathbf{2 0 0 6}$ & 199164 & $164524-233804$ & 4981576 & 4.0 \\
$\mathbf{2 0 0 7}$ & 171660 & $144749-198571$ & 5081303 & 3.4 \\
$\mathbf{2 0 0 8}$ & 129922 & $108960-150884$ & 5303406 & 2.5 \\
$\mathbf{2 0 0 9}$ & 139611 & $103660-175562$ & 4837391 & 2.9 \\
$\mathbf{2 0 1 0}$ & 195898 & $109277-282519$ & 3203046 & 6.1 \\
$\mathbf{2 0 1 1}$ & 133858 & $97327-170389$ & 5877646 & 2.3 \\
$\mathbf{2 0 1 2}$ & 86859 & $41444-132274$ & 3591527 & 2.4 \\
$\mathbf{2 0 1 3}$ & 19904 & $12778-27030$ & 4664514 & 0.4 \\
$\mathbf{2 0 1 4}$ & 2954 & $1587-4321$ & 1834175 & 0.2 \\
\hline
\end{tabular}


(CB), y la interacción año:mes fueron significativas en la explicación de la variabilidad general del modelo. La variabilidad total explicada por el modelo delta fue mayor que la del GLM, en la cual se obtuvo un $26 \%$, indicando que una proporción significativa de la variabilidad del descarte no está siendo explicada por el modelo.

Aun cuando el porcentaje de explicación es bajo, el modelo delta parece ser una mejor metodología para estimar el descarte por exceso de captura, ya que como es típico para estos casos, los datos tienen una gran proporción de observaciones que son ceros, lo cual dificulta el aplicar métodos tradicionales de estandarización para transformar los datos y que éstos cumplan con la normalidad y varianza constante requeridas. Trabajos como los de Lo et al. (1992), Pennington (1996), Stefánsson (1996), Ortiz et al. (2000) y Ortiz y Arocha (2004) demostraron que ese tipo de modelos son más consistentes con datos que tienen una gran proporción de ceros en las capturas. Sin embargo hay que tener cuidado cuando se desea aplicar el modelo delta, ya que si no se cumplen los supuestos, éste reduce grandemente su eficiencia en las estimaciones, particularmente cuando se tiene un bajo número de observaciones positivas $(\mathrm{n} \leq 40)$ o si la variable de respuesta no se acerca a la distribución normal (Myers \& Pepin 1990). Otro asunto a considerar en el modelo delta, es que el tipo de transformación generalmente usada para que la variable de respuesta presente distribución normal es el uso de logaritmos, lo cual podría causar que se obtengan números negativos, si los valores a los cuales se les aplica dicha transformación están muy cercanos a cero. Para este caso de estudio, el número de datos con valores positivos de los descartes por exceso de captura por viaje superaron las 40 observaciones, tienden a la normal luego de aplicarles la transformación box-cox (además que en dicha transformación para esos datos positivos se evita el problema de la elección de una constante c como sucedió en el modelo GLM) y los valores positivos observados no estuvieron tan cerca del cero (valores positivos $\geq 0.5$ ton).

El promedio anual del descarte por exceso de captura durante el periodo 2005 - 2014 se estimó en 121312 toneladas, el cual representó una tasa del $2.6 \%$ en relación a los desembarques. Estos porcentajes estimados se acercan a lo reportado por Vásquez y Lam (1977) quienes estimaron un porcentaje de $5 \%$ para el descarte por exceso de captura en la pesquería de anchoveta, pero significativamente menores a las estimaciones reportadas por Castillo y Mendo (1987) quienes estimaron que el descarte por exceso de captura fue de $9.13 \%$. Ambos trabajos utilizan metodologías diferentes que tal vez hace que los resultados no puedan ser comparados, sobre todo con lo reportado por Castillo y Mendo (1987) quienes basaron su metodología en encuestas a pescadores $(n=40)$ y el área de estudio no abarcó toda la distribución del stock Norte-Centro de la anchoveta peruana.

En cuanto a la variación anual de los descartes, se relacionó esta con las magnitudes de la captura total corregida por año. En ciencia pesquera generalmente supone que mientras más se captura más se descarta (Borges et al. 2004), sin embargo nuestros resultados muestran que este comportamiento no se está reflejando de manera clara (Fig. 9), por lo tanto, se podría decir que en esta pesquería no existe una proporcionalidad de las capturas con las magnitudes descartadas coincidiendo con lo reportado por Tamsett y Janacek (1999) y Trenkel et al. (2001).

En años recientes (2013-2014) el descarte por exceso de captura ha disminuido, esto coincide con un cambio en el comportamiento espacial de la flota dedicada a la extracción de anchoveta para consumo humano indirecto, las cuales durante esos años debieron pescar fuera de las 10 millas náuticas (Decreto Supremo N ${ }^{\circ}$ 005-2012-PRODUCE), además se conoce que las mayores densidades de anchoveta se encuentran cerca a la costa (aproximadamente 20 millas náuticas), sobretodo en eventos como El Nińo en el cual el recurso tiende a agregarse (Bertrand et al. 2004), ello podría ser una explicación de la disminución de las magnitudes del descarte por exceso de captura en ese periodo. Esto además indicaría que una posible variable a incluir en el modelo, para mejorar el análisis y entender el comportamiento de los descartes, sería la distancia a la costa y/o la agregación del cardumen. Otro factor a analizar es el

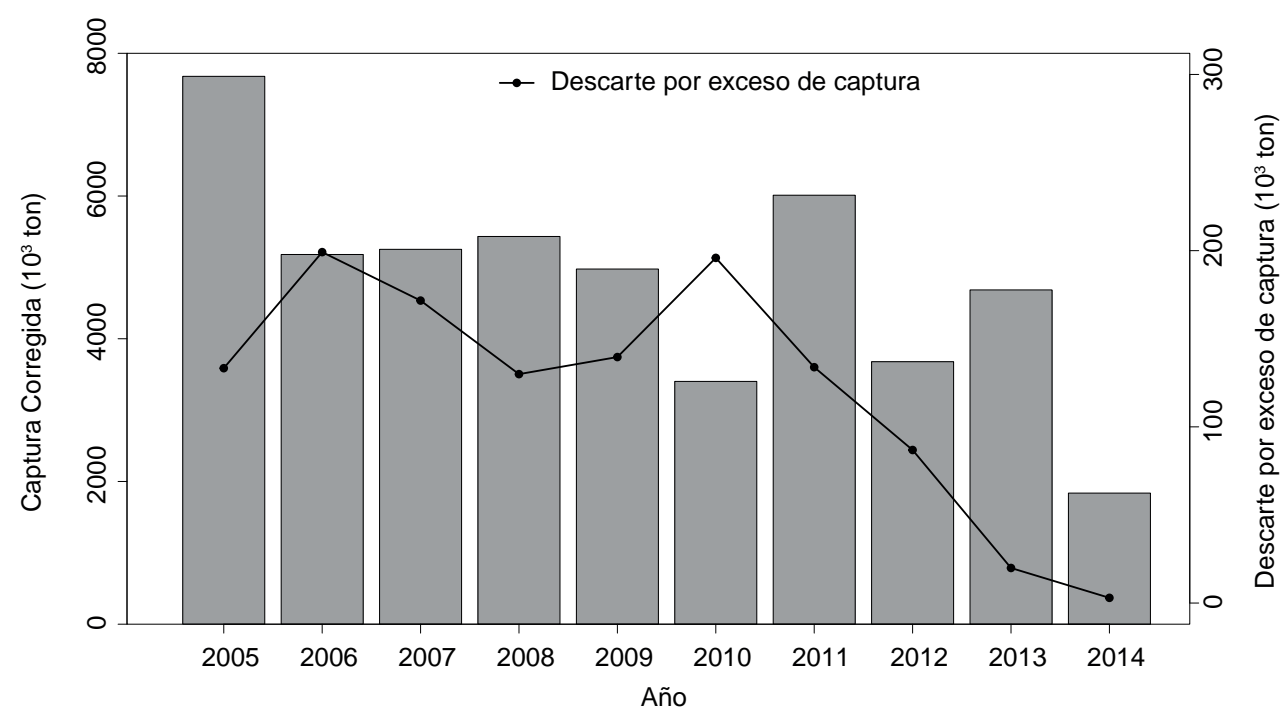

Figura 9. Captura corregida y el Descarte por exceso de captura de la pesquería industrial de cerco del stock Norte-Centro de la anchoveta peruana. 
comportamiento del pescador, pues se conoce que el descarte es un proceso decidido a bordo, basado en el comportamiento específico del pescador el cual puede estar influenciado por el tamaño de la captura, precios del mercado y/o legislaciones (Pennino et al. 2014), es así que una razón de la disminución del descarte podría deberse al comportamiento actual del pescador, ya que para él no sería necesario llenar toda su bodega con la captura, sino lo necesario para tener una mejor calidad de pesca y en consecuencia un mejor precio en la posterior venta del producto, por tanto las probabilidades de descartar por un exceso de captura disminuyen.

Este estudio muestra que la pesquería industrial de cerco dedicada a la pesca de anchoveta peruana, descarta por exceso de captura una pequeńa proporción en relación a lo que se desembarca por año, sin embargo esta no deja de ser una importante cantidad ( $\sim 121000$ ton.), la cual podría afectar la dinámica de este recurso, así como otras especies de la comunidad circundante, pues la anchoveta es la presa principal de mamíferos marinos, aves, peces convirtiéndose así en es un elemento clave de la cadena alimenticia en el sistema corriente de Humboldt (Espinoza \& Bertrand 2008). Por tanto, la incorporación de las estimaciones de los descartes a los procesos de evaluación del recurso es importante, ya que al no incluir la fracción de mortalidad por pesca que deriva de los descartes, hará que se sobreestimen las magnitudes de biomasas o abundancias. Por otro lado, incorporar dicha información proporciona una base científica para un enfoque ecosistémico de la gestión pesquera (Gislason et al. 2000), teniendo en consideración que existen otras fuentes de descarte, cuya caracterización y estimación constituirá un aporte significativo al ordenamiento pesquero de los recursos involucrados. Por ello, es necesario seguir contando con la información que proporciona el programa de observación a bordo y aumentar sus niveles de muestreo (observadores), ya que se ha demostrado que estimaciones confiables de los descartes sólo pueden ser obtenidos a partir de los datos que se colectan en estos programas (Rochet et al., 2002).

Además es importante mencionar que existen diferentes metodologías para estimar los descartes (estimación por estratificación, utilizando ponderación mediante proporciones, métodos Bayesianos). Los métodos de estimación presentados en este artículo no presentan gran complejidad, sin embargo métodos más sofisticados pueden ser implementados, aunque estos requieren un mayor conocimiento de los factores que causan la variabilidad de los descartes. El estimador apropiado en una situación dada, va a depender principalmente del uso que deba hacerse de la estimación, ya sea para incluir en las estimaciones de cuotas de captura, para comprender las causas de los descartes, o para implementar un enfoque ecosistémico de la pesca.

\section{Agradecimientos}

El autor desea expresar su agradecimiento a la Dirección General de Investigaciones en Recursos Pelágicos del IMARPE por brindar la información de descartes a través de su Programa de observadores a bordo de la flota de cerco llamado Programa Bitacoras de Pesca. A todas las personas involucradas en el desarrollo del trabajo por su valiosos comentarios. A la Dra. Rocío Joo por sus correcciones y valiosas sugerencias al inicio de la escritura de este artículo.

\section{Literatura citada}

Alverson D.L., M.H Freeberg, S.A Murawski \& J.G Pope. 1994. A global assessment of fisheries bycatch and discards. FAO Fisheries Technical Paper. No. 339. Rome, FAO. 1994. 223pp.

Babcock E.A., E.K. Pikitch \& C.G. Hudson. 2003. How much observer coverage is enough to adequately estimate by-catch? Report of the Pew Institute for Ocean Science, Rosentiel School of Marine and Atmospheric Science, University of Miami, Miami, FL. 36pp.

Bertrand A., M. Segura, M. Gutiérrez \& L. Vásquez. 2004. From smallscale habitat loopholes to decadal cycles: a habitat-based hypothesis explaining fluctuation in pelagic fish populations off Peru. Fish and fisheries, 5(4): 296-316. doi: http://dx.doi. org/10.1111/j.1467-2679.2004.00165.x

Berry D.A. 1987. Logarithmic transformations in ANOVA. Biometrics, 43(2): 439-456.

Borges L., E. Rogan, \& R. Officer. 2005. Discarding by the demersal fishery in the waters around Ireland. Fisheries Research, 76(1): 1-13. doi: http://dx.doi.org/10.1016/j. fishres.2005.05.011

Box G.E. \& D.R. Cox. 1964. An analysis of transformations. Journal of the Royal Statistical Society, Series B (Methodological), 26(2): $211-252$

Castillo S. \& J. Mendo. 1987. Estimation of unregistered Peruvian anchoveta (Engraulis ringens) in official catch statistics, 1951-1982. The Peruvian Anchoveta and Its Upwelling Ecosystem: Three Decades of Change, ICLARM studies and reviews, Manilla, Philippines, pp.109-116.

Clucas I. 1997. A study of the options for utilization of bycatch and discards from marine capture fisheries. FAO Fisheries Circular No. 928, 59pp.

Cordeiro G.M. \& C.G. Demétrio. 2008. Modelos lineares generalizados e Extensoes. Sao Paulo.

DS Nº 005-2012-PRODUCE. 2012. Modifican reglamento de ordenamiento pesquero del recurso anchoveta y anchoveta blanca, establecen zonas de reserva para consumo humano directo y régimen excepcional. 25 de agosto de 2012. El Peruano Normas Legales: 473316 - 473319.

Espinoza P. \& A. Bertrand. (2008). Revisiting Peruvian anchovy (Engraulis ringens) trophodynamics provides a new vision of the Humboldt Current system. Progress in Oceanography, 79(2), 215-227. doi: http://dx.doi.org/10.1016/j. pocean.2008.10.022.

FAO (Food and Agriculture Organization). 1995. Code of Conduct for Responsible Fisheries. Rome, 41pp. <http://www.fao.org/ docrep/005/v9878e/v9878e00.htm> (Acceso 10/05/15).

Fréon P., M. Bouchon, C. Mullon, et al. 2008. Interdecadal variability of anchoveta abundance and overcapacity of the fishery in Peru. Progress in Oceanography, 79(2): 401-412. doi: http:// dx.doi.org/10.1016/j.pocean.2008.10.011

Guerrero V.M. \& R.A. Johnson. 1982. Use of the Box-Cox transformation with binary response models. Biometrika, 69(2): 309 - 314. doi: http://dx.doi.org/10.2307/2335404.

Kelleher K. 2005. Discards in the world's marine fisheries: An update. Food and Agriculture Organization. Rome.147pp.

Lindsey J.K. 1997. Applying generalized linear models. Springer Science \& Business Media. 256pp.

Lo N.C., L.D. Jacobson \& J.L. Squire. 1992. Indices of Relative Abundance from Fish Spotter Data based on Delta-Lognormal Models. Canadian Journal of Fisheries and Aquatic Sciences, 49(12): 2515 - 2526. doi: http://dx.doi.org/10.1139/ f92-278.

Machias A., V. Vassilopoulou, D. Vatsos, et al. 2001. Bottom trawl discards in the northeastern Mediterranean Sea. Fisheries Research, 53(2): 181 -195. doi: http://dx.doi.org/10.1016/ S0165-7836(00)00298-8.

McCullagh P. 1984. Generalized linear models. European Journal of Operational Research, 16(3): 285 - 292. doi: http://dx.doi. org/10.1016/0377-2217(84)90282-0.

Myers R. \& P. Pepin. 1990. The robustness of lognormal-based estimators of abundance. Biometrics, 46(4): 1185 - 1192. doi: http://dx.doi.org/10.2307/2532460. 
Ortiz M., C. Legault, N. Ehrhardt. 2000. An alternative method for estimating bycatch from the US shrimp trawl fishery in the Gulf of Mexico, 1972-1995. Fishery Bulletin, 98(3): $583-589$.

Ortiz M. \& F. Arocha. 2004. Alternative error distribution models for standardization of catch rates of non-target species from a pelagic longline fishery: billfish species in the Venezuelan tuna longline fishery. Fisheries Research, 70(2): 275-297. doi: http://dx.doi.org/10.1016/j.fishres.2004.08.028

Pennington M. 1983. Efficient estimators of abundance, for fish and plankton surveys. Biometrics, 39: 281 - 286. doi: http:// dx.doi.org/10.2307/2530830

Pennington M. 1996. Estimating the mean and variance from highly skewed marine data. Fishery Bulletin, 94(3): $498-505$.

Rochet M.J., I. Péronnet \& V.M. Trenkel. 2002. An analysis of discards from the French trawler fleet in the Celtic Sea. ICES Journal of Marine Science: Journal du Conseil, 59(3): 538-552. doi: http://dx.doi.org/10.1006/jmsc.2002.1182.

Saila S.B. 1983. Importance and assessment of discards in commercial fisheries. Food and Agriculture Organization, Fisheries Circular $\mathrm{N}^{\circ} 765$, Rome, FAO.

Sakia R. 1992. The Box-Cox transformation technique: a review. The statistician. 41(2): 169 - 178. doi: http://dx.doi. org $/ 10.2307 / 2348250$
Sprugel D.G. 1983. Correcting for bias in log-transformed allometric equations. Ecology, 64(1), 209-210. doi: http://dx.doi. org/10.2307/1937343.

Spitzer J.J. 1982. A primer on Box-Cox estimation. The Review of Economics and Statistics. 64(2): 307-313. doi: http://dx.doi. org/10.2307/1924310.

Steffansson G. 1996. Analysis of ground fish survey abundance data: combining the GLM and delta approaches. ICES Journal of Marine Science: Journal du Conseil, 53(3): 577-588. doi: http://dx.doi.org/10.1006/jmsc.1996.0079.

Tamsett D. \& G. Janacek. 1999. Sampling trips for measuring discards in commercial fishing based on multilevel modelling of measurements in the North Sea from NE England. Fisheries Research, 42(1): 103-115. doi: http://dx.doi.org/10.1016/ S0165-7836(99)00032-6

Trenkel V.M., M.J. Rochet \& M. Joelle. 2001. Towards a theory for discarding behaviour. ICES CM, 3: 10.

Viana M. 2012. Temporal and spatial dynamics of fisheries discards from Irish waters. Thesis of Doctor of Philosophy. University of Dublin-Ireland, Trinity College. <http://www.tcd.ie/ Zoology/research/research/theoretical/Viana/Mafalda\%20 Viana\%20PhD\%20thesis\%20-\%20Discards\%20-\%20 PhD-FS-08-001-01.pdf > Acceso 30/07/2015.

Yamamura K. 1999. Transformation using $(x+0.5)$ to stabilize the variance of populations. Researches on Population Ecology, 41(3): 229-234. doi: http://dx.doi.org/10.1007/ s101440050026. 